\title{
Ein Leben für die Musikpsychologie: Nachruf Prof. Dr. Maria Manturzewska (1930 - 2020)
}

\author{
A Life for Music Psychology: Obituary Prof. Dr. Maria Manturzewska (1930 - 2020)
}

Heiner Gembris*a

[a] Institut für Begabungsforschung in der Musik (IBFM), Universität Paderborn, Paderborn, Deutschland.

\section{Zusammenfassung}

Dieser Beitrag würdigt die Lebensleistung der polnischen Musikpsychologin Prof. Dr. Maria Manturzewska (1930 - 2020) für die polnische und internationale Musikpsychologie. Sie gründete und leitete bereits 1956 die erste Arbeitsgruppe für musikpsychologische Forschung am Pädagogischen Zentrum der Kunst- und Musikschulen des Kultusministeriums in Warschau und veranstaltete die erste musikpsychologische Konferenz in Polen. In den folgenden Jahren importierte sie alle verfügbaren standardisierten Musiktests der damaligen Zeit sowie Intelligenz- und Persönlichkeitstests, die in einem Netzwerk von Psychologen übersetzt, restandardisiert und normiert wurden. Dieses Instrumentarium wurde sowohl zur Erforschung musikalischer Begabung und Entwicklung eingesetzt als auch in der psychologischen Beratung im Rahmen der Begabungsförderung. Mit ihren wegweisenden Forschungen zur biographischen Entwicklung von professionellen Musiker*innen in den 1970er Jahren hat sie der Etablierung der Lebenszeitperspektive musikalischer Entwicklung entscheidende Impulse gegeben. Mit der Gründung des Instituts für Forschung in der Musikpädagogik 1974 an der Chopin Musik Akademie Warschau hat sie einen bedeutenden Meilenstein für die Institutionalisierung der Musikpsychologie erreichen können. 1985 erhielt sie dort den ersten Lehrstuhl für Musikpsychologie in Polen, den sie bis zu ihrer Pensionierung im Jahr 2000 innehatte. Neben ihren wegweisenden Forschungen zur musikalischen Begabung und zur lebenszeitlichen musikalischen Entwicklung hat Maria Manturzewska sich um die Entwicklung, Etablierung und Institutionalisierung der Musikpsychologie verdient gemacht. Unter ihrer Führung wurde die polnische Musikpsychologie in den Nachkriegsjahren aufgebaut, über Jahrzehnte weiterentwickelt und zu einer bemerkenswerten Blüte und internationalen Wertschätzung gebracht, die eng mit ihrer Person verbunden ist. Das Lebenswerk Maria Manturzewskas wird für die wissenschaftliche Gemeinschaft auch in Zukunft eine Quelle wissenschaftlicher Erkenntnis und Inspiration sein.

Schlüsse/wörter: Musikpsychologie, Polen, musikalische Begabung, Begabungsdiagnostik, Musikalitätstest, musikalische Entwicklung, Begabungsförderung, Musikpädagogik, Lebenszeitperspektive musikalischer Entwicklung

\begin{abstract}
This paper pays tribute to the lifetime achievements of the Polish music psychologist Prof. Dr. Maria Manturzewska (1930 - 2020) for Polish and international music psychology. She founded and headed the first working group for music psychology research at the Pedagogical Center of Art and Music Schools of the Ministry of Culture in Warsaw as early as 1956 and organized the first music psychology conference in Poland. She imported all available standardized music tests of that time, as well as intelligence and personality tests, which were translated, restandardized, normalized and applied in research and music education. With her pioneering research on the biographical development of professional musicians, she gave decisive impulses to the establishment of the lifetime perspective of musical development. With the founding of the Institute for Research in Music Education in 1974 at the Chopin Academy of Music in Warsaw, she was able to achieve a significant milestone for the institutionalization of music psychology. In 1985, she was appointed to the first chair of music psychology in Poland, which she held until her retirement in 2000. In addition to her pioneering research on musical talent and lifespan musical development, Maria Manturzewska was outstanding in the development, establishment, and institutionalization of music psychology. Under her leadership, Polish music psychology was established in the postwar years, and brought to a remarkable flowering and international recognition closely associated with her person. Maria Manturzewska's life's work will continue to be a source of scientific insight and inspiration for the scientific community.
\end{abstract}

Keywords: music psychology, Poland, musical giftedness and talent, measurement of giftedness, psychometric research, music education, gifted education, musical development, lifespan perspective of musical development 
Jahrbuch Musikpsychologie, 2021, Vol. 30: Musikpsychologie - Empirische Forschungen - Ästhetische Experimente, Artikel e85, https://doi.org/10.5964/jbdgm.85

Publiziert (VoR): 2021-02-16. Korrigiert (CVoR): 2021-02-18.

*Korrespondenzanschrift: Institut für Begabungsforschung in der Musik (IBFM), Universität Paderborn, Pohlweg 85, 33100 Paderborn, Deutschland. EMail: heiner.gembris@uni-paderborn.de

Dieser Open-Access-Artikel steht unter den Bedingungen einer Creative Commons Namensnennung 4.0 International Lizenz, CC

BY 4.0 (https://creativecommons.org/licenses/by/4.0/deed.de). Diese erlaubt für beliebige Zwecke (auch kommerzielle) den Artikel zu verbreiten, in jedwedem Medium zu vervielfältigen, Abwandlungen und Bearbeitungen anzufertigen, unter der Voraussetzung, dass

der Originalartikel angemessen zitiert wird.

Maria Manturzewska, die Grande Dame der polnischen Musikpsychologie, ist am 11. November $2020 \mathrm{im}$ Alter von 90 Jahren in Warschau verstorben. Geboren am 30. Juni 1930 in Krakau, wuchs sie in einem musikalischen Umfeld auf und studierte an der Jagiellonen-Universität Krakau, einer der ältesten Universitäten Europas, die Fächer Psychologie, Philosophie und Anthropologie. Zu ihren akademischen Lehrern zählten Stefan Szumann, ihr späterer Doktorvater, der die Polnische Schule der Entwicklungspsychologie mitbegründete und bereits Ende der 1930er Jahre an der Entwicklung von musikalischen Begabungstests forschte, sowie der Philosoph Roman Imgarden, der einflussreiche Werke zur Philosophie der Kunst und zur Ästhetik schuf. In diesem inspirierenden akademischen Umfeld entstand ihre Masterarbeit (1952) zur Entwicklung der Wahrnehmungsfähigkeiten des emotionalen Ausdrucks in der Musik bei Kindern und Jugendlichen, mit der sie bereits einen späteren Schwerpunkt ihrer wissenschaftlichen Arbeit in der musikalischen Entwicklungspsychologie markierte.

Im Jahr 1953 übersiedelte Maria Manturzewska mit ihrem Mann nach Warschau, wo sie zunächst bis 1956 am Museum für Volkskultur (heute Staatliches Ethnographisches Museum Warschau) arbeitete. Gleichzeitig wirkte sie an interdisziplinären Forschungen zur Musikwahrnehmung an der Warschauer Musikhochschule (heute Chopin University of Music) mit. Zwischen 1956 und 1961 arbeitete sie am Pädagogischen Zentrum Künstlerischer Schulen, einer Einrichtung des Kultusministeriums, die für die Entwicklung der staatlichen Kunst- und Musikschulen zuständig war (vgl. Kotarska, 1986). Dort gründete sie 1956 die erste Arbeitsgruppe für Musikpsychologie. In diesem Zusammenhang führte sie bereits im Dezember 1956 die erste Musikpsychologische Konferenz in Polen durch, durch die sie musikpsychologisch Interessierte von Universitäten, Musikhochschulen und Musikschulen, namhafte Musiker*innen und Medienvertreter*innen zusammenbrachte.

Ein wichtiger Schritt der neugegründeten Forschungseinrichtung für Musikpsychologie war der Import aller damals international verfügbaren standardisierten Tests zu Messung musikalischer Begabung bzw. Leistung, um sie hinsichtlich ihrer Anwendbarkeit und Nützlichkeit für polnische Schulkinder zu überprüfen (vgl. Kotarska, 1986; Manturzewska, 1992, S. 17). Als Teil des Projekts wurden auch die Methoden und Praktiken der Bewertung musikalischer Begabung in den Musikschulen erforscht sowie Musiklehrer*innen durch Informationen und Vorlesungen mit wissenschaftlichen Methoden der Begabungsmessung vertraut gemacht. Hintergrund dieser sehr praxisverbundenen Forschungsaktivitäten war, dass die Dropout-Rate an den leistungsorientierten polnischen Musikschulen trotz anspruchsvoller Aufnahmeverfahren sehr hoch war. Die Adaption und Entwicklung von standardisierten Testverfahren zur Diagnose von musikalischer Begabung und Leistung sollten dazu beitragen, die Aufnahmekriterien an Musik(hoch)schulen zu objektivieren und zu verbessern. In Jahren intensi- 
ver Forschung in einem Netzwerk von Expertenteams erfolgte die Adaption, Restandardisierung, Normierung und Veröffentlichung verschiedener internationaler Musik-Tests (u. a. Wing's Standardised Tests of Musical Intelligence, Drake's Musical Memory Test, Farnum's Music Notation Test) sowie von standardisierten Persönlichkeitstests (z. B. Eysenck's Maudsley Personality Inventory und Cattell's Self-Analysis Form) in polnischer Sprache. Sie dienten nicht nur der Messung musikalischer Begabung und Leistung, sondern auch der Untersuchung von Zusammenhängen mit Persönlichkeitsmerkmalen (vgl. Manturzewska, 1978, 1992).

Anfang der 1960er Jahre wandte Maria Manturzewska sich der Untersuchung der Teilnehmer*innen des 6. Internationalen Warschauer Chopin Klavierwettbewerbs zu. Im Vordergrund dieses Projektes stand die Erforschung der Begabung, Intelligenz, Persönlichkeit und Interessen der Teilnehmer*innen anhand von Fragebögen und psychologischen Tests. Auf der Basis dieser Studien erfolgte ihre Promotion über psychologische Bedingungen hoher Leistungen von Pianisten (1963, veröffentlicht als Manturzewska, 1969). Dies war nicht nur ein Meilenstein in ihrer beruflichen Karriere, sondern auch für die Musikpsychologie als Fachdisziplin, denn es handelte sich um die erste musikpsychologische Promotion in Polen. Im Kontext ihrer Studien zum 6. Internationalen Klavierwettbewerb in Warschau betrat Maria Manturzewska wiederum musikpsychologisches Neuland mit einer weiteren, wegweisenden Studie, in der sie die Reliabilität der Urteile der Jury des 6. Internationalen Chopin Wettbewerbs anhand von Tonbandaufnahmen der Wettbewerbsbeiträge in experimentellen Settings überprüfte.

In den Jahren 1967 bis 1972 untersuchte sie den Einfluss von persönlichkeitsspezifischen, biographischen und pädagogischen Faktoren für Erfolg in der musikalischen Ausbildung von Musikschulschüler*innen. Mit dieser Studie zu psychologischen Determinanten des Erfolgs in der musikalischen Ausbildung wurde sie 1974 habilitiert (Manturzewska, 1974). Bis Ende der 1960er Jahre arbeitete sie als Abteilungsleiterin am Psychometrischen Labor der Polnischen Akademie der Wissenschaften. Sie organisierte auf nationaler Ebene Arbeitsgruppen zur Erforschung musikalischer Begabung, in denen Vertreter*innen von Musikhochschulen und Universitäten interdisziplinären Austausch zwischen Wissenschaft und Praxis pflegten.

Seit 1972 wirkte sie an der Warschauer Chopin Musikakademie (heute Chopin University of Music), wo sie u. a. ein Institut für Forschung in der Musikpädagogik etablierte, theoretisch fundierte psychologische Beratungskonzepte für Musikstudierende entwickelte und in der praktischen Beratung von Studierenden und Musiker*innen einsetzte. An der Chopin Musikakademie erhielt sie 1985 den ersten Lehrstuhl für Musikpsychologie in Polen, den sie bis zu ihrer Pensionierung im Jahr 2000 innehatte. In den 1970er Jahren führte Maria Manturzewska im Sinne des Wortes zukunftsweisende biographische Studien zur lebenszeitlichen Entwicklung von über 160 professionellen Musiker*innen durch. Sie erarbeitete u. a. ein allgemeines Entwicklungsmodell, das erstmals auf einer breiten Datenbasis verschiedene Phasen, Übergänge und Krisen in der Karriere professioneller Musiker*innen detailliert beschreibt und bis heute grundlegend ist (Manturzewska, 1990).

Neben ihren vielseitigen Tätigkeiten in Forschung und Lehre hat Maria Manturzewska unermüdlich durch ihre organisatorischen, administrativen und beratenden Tätigkeiten auf verschiedenen Ebenen an der Vernetzung von Psychologie, Musikpraxis und Musikpädagogik sowie an der Etablierung der Musikpsychologie als Fach gearbeitet. Als Mitglied der Polnischen Psychologischen Gesellschaft brachte sie die Musikpsychologie in die Konferenzen der Psychologischen Gesellschaft ein und wirkte in zahlreichen Expertengruppen mit.

Ohne Zweifel hat Maria Manturzewska nicht nur durch ihre Forschungen bahnbrechende Leistungen sowohl für die polnische als auch für die internationale Musikpsychologie geleistet. Bereits in der Nachkriegszeit der 
1950er Jahre und in den folgenden Jahrzehnten des Kalten Krieges initiierte und förderte sie durch ihr Wirken die Rezeption amerikanischer und westeuropäischer Musikpsychologie in Polen, was unter den historischen, politischen und gesellschaftlichen Bedingungen dieser Zeit in den Ländern des damaligen Ostblocks alles andere als selbstverständlich war. Sie stand in Kontakt mit Herbert Wing, dessen Tests der musikalischen Begabung eine wichtige Grundlage für ihre Forschungen waren, wie auch mit der britischen Musikpsychologin Rosamund Shuter-Dyson, deren Buch „The psychology of musical ability“ (Shuter-Dyson \& Gabriel, 1982) 1986 in einer polnischen Übersetzung erscheinen konnte. Schon bald nach dem Ende der Ost-West-Trennung Europas durch den Eisernen Vorhang im Jahr 1989 führte sie September 1990 in Radziejowice die erste Internationale musikpsychologische Konferenz in Polen durch, an der sowohl Forscher*innen aus verschiedenen Ländern Westeuropas, den USA und Japan als auch Wissenschaftler*Innen aus osteuropäischen Ländern sowie der damaligen Sowjetunion teilnahmen. Eine Dokumentation dieses „International Seminar of the Researchers and Lecturers in the Psychology of Music" wurde 1995 veröffentlicht (Manturzewska, Miklaszewski, \& Białkowski, 1995).

Die Rezeption ihrer umfänglichen Forschung und der von erfolgreich betriebenen Institutionalisierung und Vernetzung der Musikpsychologie in Polen war in den Ländern des Westens über Jahrzehnte nur eingeschränkt möglich, weil die Publikation ihrer wissenschaftlichen Arbeiten meist in polnischer Sprache erfolgte und der internationale Austausch mit den Ländern des damaligen Ostblocks vor 1989 oft gar nicht oder nur unter Schwierigkeiten gelang. Ende der 1970er Jahre hat sie meines Wissens erstmals in zwei international publizierten Artikeln über die testbasierten Forschungen ihres Warschauer Musikpsychologischen Labors zur musikalischen Begabung und Entwicklung (Manturzewska, 1978) sowie über ihre Untersuchungen zu den Determinanten von musikalischer Hochleistung und Erfolg von Pianisten berichtet (Manturzewska, 1979). Der Internationale Kieler-Woche-Kongress 1985 unter dem Titel „Musikalische Begabung finden und fördern“ bot Maria Manturzewska und ihren Kolleg*innen eine der seltenen Gelegenheiten, ihre Forschungen zur musikalischen Begabung und Entwicklung vor einem internationalen Fachpublikum aus West- und Osteuropa darzustellen (Kotarska, 1986; Manturzewska, 1986; Manturzewska \& Kotarska, 1986).

Mit der Öffnung der Grenzen Osteuropas ab 1989 eröffneten sich neue Möglichkeiten der persönlichen Begegnung und des Wissenstransfers. Auf einer internationalen Expertenkonferenz zum Thema „Musikalische Hochbegabung: Findung und Förderung“ (November 1990) gab Maria Manturzewska einen Überblick über „35 Jahre Forschung und Erfahrung in der Findung und Förderung musikalischer (Hoch-) Begabung in Polen“ (Manturzewska, 1991). Ich hatte das große Glück, sie zur 9. Jahrestagung der Deutschen Gesellschaft für Musikpsychologie 1993 in Münster („Musikalische Entwicklung in der Lebenszeitperspektive“) sowie 2002 zur Tagung „Musikalische Begabung in der Lebenszeitperspektive“ des Instituts für Begabungsforschung in der Musik (IBFM) der Universität Paderborn als Referentin einladen zu können (Manturzewska, 2006). Auch über ihre Pensionierung im Jahr 2000 hinaus hat sie die Entwicklung der Musikpsychologie mit wachem Interesse verfolgt und unterstützt. Zuletzt hat sie als 88-Jährige zusammen mit Jane Ginsborg, Richard Parncutt und John Sloboda im Ehrenkommittee der 2018 in Kattowitz ausgetragenen „Conference of Music Psychology PsychoMuzy“ mitgewirkt (http://www.psychomuzy.us.edu.pl/committees/).

Als ich sie im Oktober 2018 in Warschau besuchte, hat sie ein kleines Treffen mit Vertreter*innen der polnischen Musikpsychologie aus unterschiedlichen Landesteilen arrangiert, um die Möglichkeit zu einem Austausch über die Situation der Musikpsychologie in Polen und Deutschland zu nutzen. 
Maria Manturzewska war eine bedeutende, brillante polnische Musikpsychologin von internationalem Rang. Sie knüpfte in den 1950er Jahren an eine polnische Tradition musikalischer Begabungsforschung und Testentwicklung an, die durch den 2. Weltkrieg, die deutsche Besatzung und durch ein stalinistisches Regime unterbrochen wurde. Mit ihrem Enthusiasmus, ihrem unermüdlichen Engagement, ihrer fachlichen Kompetenz und Weitsicht hat sie die Musikpsychologie in Polen über Jahrzehnte aufgebaut und geprägt, ihr entscheidende Impulse gegeben und zu internationaler Wahrnehmung und Geltung geführt. Indem sie seit Mitte der 1950er Jahre erfolgreich daran gearbeitet hat, die Musikpsychologie als empirische, interdisziplinär arbeitende Fachdisziplin auf nationaler Ebene zu begründen und zu institutionalisieren, indem sie der musikalischen Begabungs- und Entwicklungsforschung maßgebliche, innovative Impulse gab und die Lebenszeitperspektive musikalischer Entwicklung in einem großen Entwurf bereits in den 1970er Jahren auf die Agenda der Musikpsychologie setzte, war sie ihrer Zeit weit voraus.

Sie hat beharrlich die Wichtigkeit von Kooperation betont und Brücken gebaut in vielerlei Hinsicht: in der wissenschaftlich-interdisziplinären Zusammenarbeit über die Fächergrenzen hinaus, in der Kooperation von Wissenschaft, Schule, Eltern und Entwicklungsberatung; in der Kommunikation von Wissenschaft in die Praxis; in der internationalen Zusammenarbeit, die auch politische Grenzen überwunden und Menschen aus Ost und West zusammengeführt hat in Zeiten, in denen das weder einfach noch selbstverständlich gewesen ist. Sie war eine Persönlichkeit von profunder Bildung, verbunden mit herzlicher Wärme und Zugewandtheit, ein Familienmensch, eine Freundin und Vorbild. Ihr Werk wird in der Musikpsychologie weiterleben, uns in Zukunft weiter begleiten und inspirieren. Danke, Maria.

\section{Danksagung}

Ich danke Dr. Kacper Miklaszewki (Warschau) und Dr. Stella Kaczmarek (Lodz) für ihre freundliche Unterstützung bei der Recherche von biographischen Informationen und Quellen.

\section{Literatur}

Kotarska, H. (1986). Entwicklungstendenzen und Leistungen der musikpsychologischen Diagnose in Polen. In E. Rohlfs, E.

Auer \& H. Damsch (Hrsg.), Musikalische Begabung finden und fördern: Materialien und Dokumente Kieler-Woche-

Kongreß 1985 (S. 93-95). Regensburg, Deutschland: Bosse.

Manturzewska, M. (1969). Psychologiczne Warunki Osiągnięć Pianistycznych [Psychological conditions of piano achievement]. Wroclaw, Polen: PAN.

Manturzewska, M. (1974). Psychologiczne wyznaczniki powodzenia w studiach muzycznych [Psychological determinants of success in music studies]. Warszawa, Polen: COPSA.

Manturzewska, M. (1978). Psychology in the music school. Psychology of Music, 6(2), 36-47.

https://doi.org/10.1177/030573567862004

Manturzewska, M. (1979). Results of psychological research on the process of music practicing and its effective shaping. Bulletin of the Council for Research in Music Education, 59, 59-61. 
Manturzewska, M. (1986). Musikalisches Talent im Lichte biographischer Forschung. In E. Rohlfs, E. Auer \& H. Damsch (Hrsg.), Musikalische Begabung finden und fördern: Materialien und Dokumente Kieler-Woche-Kongreß 1985 (S. 79 85). Regensburg, Deutschland: Bosse.

Manturzewska, M. (1990). A biographical study of the life-span development of professional musicians. Psychology of Music, 18(2), 112-139. https://doi.org/10.1177/0305735690182002

Manturzewska, M. (1991). 35 Jahre Forschung und Erfahrung in der Findung und Förderung musikalischer (Hoch-) Begabung in Polen. In H.-G. Bastian (Hrsg.), Musikalische Hochbegabung: Findung und Förderung: Dokumentation einer Internationalen Expertenkonferenz (S. 163-172). Mainz, Deutschland: Schott.

Manturzewska, M. (1992). Identification and promotion of musical talent. European Journal for High Ability, 3(1), 15-27. https://doi.org/10.1080/0937445920030102

Manturzewska, M. (2006). A biographical study of the lifespan development of professional musicians. In H. Gembris (Hrsg.), Musical development from a lifespan perspective (S. 21-53). Frankfurt, Deutschland: Lang.

Manturzewska, M., \& Kotarska, H. (1986). Musikalische Fähigkeiten im Licht psychometrischer Forschung. In E. Rohlfs, E. Auer \& H. Damsch (Hrsg.), Musikalische Begabung finden und fördern: Materialien und Dokumente Kieler-WocheKongreß 1985 (S. 99-104). Regensburg, Deutschland: Bosse.

Manturzewska, M., Miklaszewski, K., \& Białkowski, A. (Hrsg.). (1995). Psychology of music today: Proceedings of the Internatiional Seminar of Researchers and Lecturers in the Psychology of Music, Radziejowice, Poland, 24-29 September 1990. Warsaw, Polen: Fryderyk Chopin Academy of Music.

Shuter-Dyson, R. \& Gabriel, C. (1982). The psychology of musical ability (2. Aufl.). London, United Kingdom: Methuen. (in Polnisch erschienen unter: Psychologia uzdolnienia muzycznego, Wydanie: WSiP 1986. Warszawa, Polen: Wydawnictwa Szkolne i Pedagogiczne). 